\title{
Stormy Weather and Cluster Radio Galaxies
}

\author{
J. O. Burns ${ }^{\mathrm{a}, 1}$, C. Loken ${ }^{\mathrm{a}}$, K. Roettiger ${ }^{\mathrm{a}}$, E. Rizza ${ }^{\mathrm{a}}$, \\ G. Bryan ${ }^{b}$, M. L. Norman ${ }^{\mathrm{c}}$, P. Gómez ${ }^{\mathrm{d}}$, F. N. Owen ${ }^{\mathrm{e}}$ \\ ${ }^{a}$ Department of Physics 8 Astronomy and Office of Research, University of \\ Missouri, Columbia, MO 65211 \\ ${ }^{\mathrm{b}}$ Department of Physics, M.I.T., Cambridge, MA 02139 \\ ${ }^{\mathrm{c}}$ Department of Astronomy, University of Illinois, Urbana, IL 61801 \\ ${ }^{\mathrm{d}}$ Department of Physics $\&$ Astronomy, Rutgers University, Piscataway, NJ 08855 \\ e National Radio Astronomy Observatory, P. O. Box O, Socorro, NM 87801
}

\begin{abstract}
New adaptive mesh refinement N-body + hydrodynamics numerical simulations are used to illustrate the complex and changing cluster environments in which many radio galaxies live and evolve. Groups and clusters of galaxies form at the intersections of filaments where they continue to accrete gas and dark matter to the present day. The accretion process produces shocks, turbulence, and transonic bulk flows forming a kind of stormy weather within the intracluster medium (ICM). Radio sources embedded within the stormy ICM form distorted, complex morphologies as observed in recent VLA cluster surveys. We show that the bending of wide-angle tailed radio sources can be understood as the result of recent cluster-subcluster mergers. We use new MHD simulations to illustrate how cluster radio halos can be formed by the shocks and turbulence produced during cluster mergers. Finally, we discuss new observations of distant Abell clusters that reveal a class of weak radio sources, probably starbursts, likely produced during the formation of the clusters as they accrete material from the supercluster environment.
\end{abstract}

\section{The Environments Within Which Radio Galaxies Live \& Evolve}

The environments of radio galaxies change dramatically over time. Galaxies form within a large-scale cosmic web that contains $>100$ Mpc-length filaments, composed of dark and baryonic matter, and huge voids of comparable

$\overline{1}$ E-mail: burnsj@missouri.edu

Preprint submitted to Elsevier Preprint 23 September 2018 
size. Groups and clusters, where nearly all galaxies reside, form at the intersections of filaments. Clusters continue to evolve even at the present epoch by accreting gas and galaxies from connecting filaments and by merging with other clusters/groups (roughly every few Gyrs).

In Fig. 1, we illustrate the evolution of the gaseous environs of two Comalike rich clusters using a newly developed adaptive mesh refinement (AMR) numerical simulation (Loken et al. 1999). This simulation is for a $\Lambda$ CDM closed universe. The AMR code uses a series of grids that adaptively refine to higher resolution in proportion to the local density. Thus, high spatial dynamic range (8000:1) and excellent resolution in the cluster core $(\approx 16 \mathrm{kpc})$ are achieved. The simulations reveal complex evolution within groups and clusters. For example, the total cluster mass and gas density doubles between $z=0.5$ and $z=0$ as a result of on-going mergers and accretion from filaments. The dark matter density profile maintains a general power-law form (e.g. Navarro, Frenk, \& White 1997) whereas the gas profile has a definite core (well-fit by a $\beta$-model) produced from shock-heating and expansion of the central region. Clusters have substantial asymmetric temperature and pressure structure even at $z=0$; such predicted temperature substructure will soon be tested with new observations from the Chandra and XMM satellites. These simulations, along with earlier numerical models (e.g. Roettiger, Burns, \& Loken 1996), show that substantial bulk motions with velocities $\geq 1000 \mathrm{~km} / \mathrm{sec}$ can be produced from the accretion and mergers in clusters (see Fig. 2).

These simulations suggest that the ICM within groups and clusters is stormy, violent, filled with shocks, high winds, and turbulence. The low density plasmas associated with extended radio sources embedded within groups and clusters will act as windsocks and barometers of this cluster weather (Burns 1998).

\section{Wide-Angle Tailed Radio Galaxies}

Wide-angle tails (WAT) are $\mathrm{V}$-shaped, large diameter $\left(0.2-1.2 h_{75}^{-1} \mathrm{Mpc}\right)$ radio sources most often associated with $\mathrm{D} / \mathrm{cD}$ or giant ellipticals at the optical centers of clusters. They lie at the FR I/II interface. Multifiber spectroscopy has confirmed that the WAT galaxies are generally moving very slowly $(<100$ $\mathrm{km} / \mathrm{sec}$ ) relative the cluster velocity centroid (Pinkney et al. (1993), Pinkney et al. (1999)). Such slow motion is insufficient to bend the jets/tails of WATs to their observed curvature and, thus, an interesting puzzle emerges concerning the origin of the shape of these relatively powerful cluster radio sources (Eilek et al. 1984).

Following the launch of ROSAT, we observed a statistical sample of WAT clusters using the PSPC detector with $\approx 30$ " resolution (Gómez et al. 1997). 

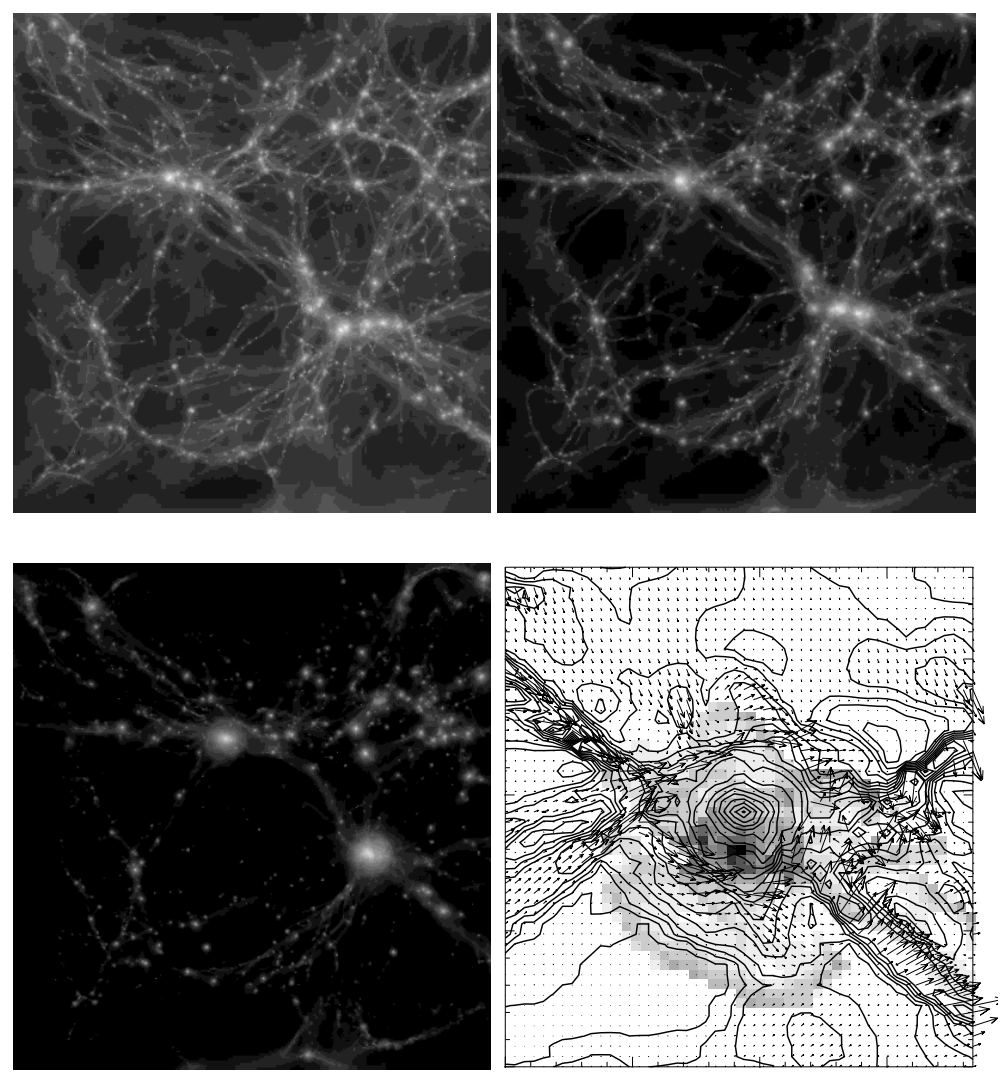

Fig. 1. AMR simulation of the evolution of rich clusters of galaxies in a $\Lambda \mathrm{CDM}$ universe. The top two panels $(z=1$ and $z=0.5)$ and bottom left panel $(z=0)$ display the evolution of gas density within a subvolume with sides of length $\sim 50 \mathrm{~h}^{-1}$ Mpc. The bottom right panel shows gas density contours, momentum vectors (below a minimum threshold), and grey-scale temperature (for gas between $10^{6}$ and $10^{8} \mathrm{~K}$ ) in a $20 \mathrm{~h}^{-1} \mathrm{Mpc}$ region centered on the largest of the two $z=0$ clusters. The entire computational volume has sides of $256 \mathrm{~h}^{-1} \mathrm{Mpc}$. The mass resolution is $\sim 10^{10} \mathrm{M}_{\odot}$ and the spatial resolution is $\sim 15.6 \mathrm{~h}^{-1} \mathrm{kpc}$.

These x-ray images revealed statistically significant substructure in $90 \%$ of the WAT clusters. Furthermore, this substructure generally consists of x-ray elongations that align closely with the directions of the radio tails.

In Fig. 2, we compare x-ray/radio observations of a WAT cluster with the results of an N-body/hydro simulation of a cluster undergoing a merger with a smaller group of galaxies (Roettiger, Burns, \& Loken 1996). These simulations strongly suggest that the WAT tails are bent by motion of the ICM past the radio galaxies arising from bulk flows following the merger between clusters. This contrasts with the traditional model of bending tailed radio sources via motion of the radio galaxy through a static ICM.

This model has found additional support from the recent observation that there is a strong alignment between the directions of WAT tails and the local supercluster axis as defined by the distribution of nearby Abell clusters 

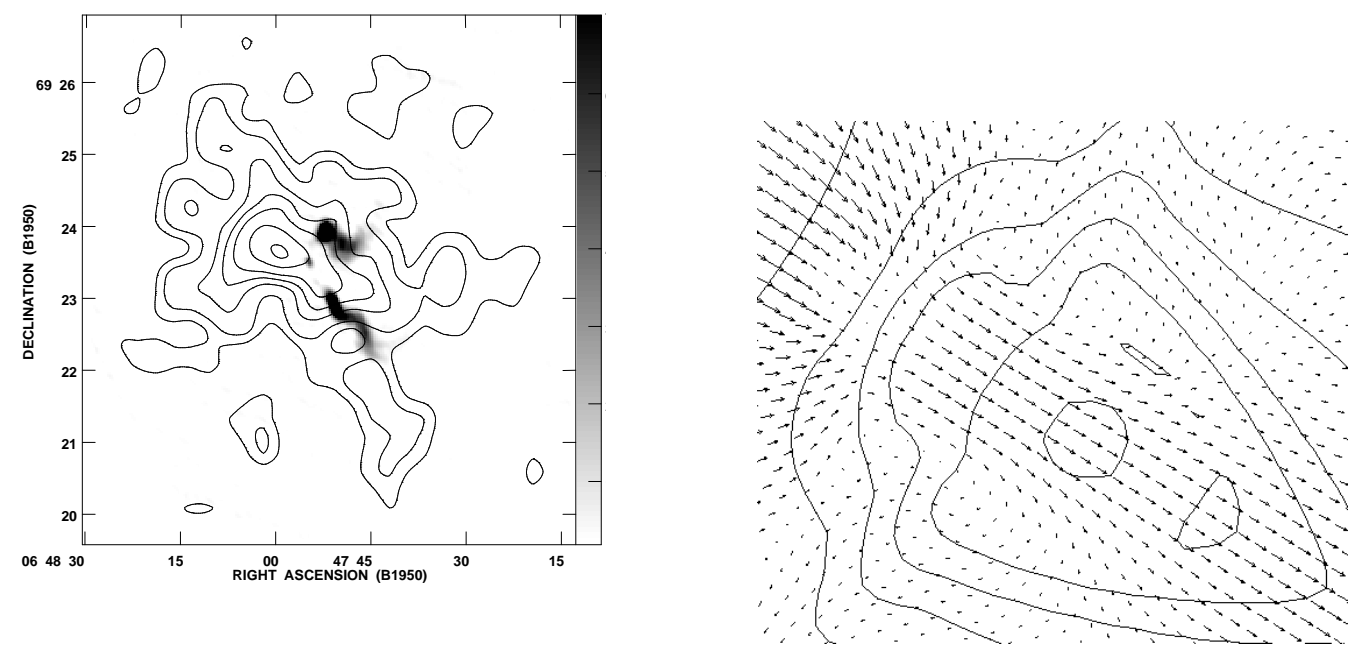

Fig. 2. Left: Overlay of $6 \mathrm{~cm}$ greyscale radio map onto a ROSAT PSPC image of A562. Right: Synthetic x-ray contours and velocity vectors from a cluster-subcluster simulation with a 4:1 mass ratio at 0.5 Gyrs after the subcluster passed through the cluster core. Field is $2 h_{75}^{-1} \mathrm{Mpc}$ on a side with the longest vector of $1941 \mathrm{~km} / \mathrm{sec}$. Note the bulk flow is along the merger axis and coincides with the x-ray elongation.

(Novikov et al. 1999). Thus, we believe that the large scale environment (i.e., accretion from filaments) is having an important direct impact on the central regions, including radio sources, within clusters.

\section{Radio Halos and Cluster Mergers}

Cluster radio halos are relatively rare (Giovannini et al. (1999) identify 29 candidate halo/relic souces), have steep radio spectra $(\alpha \geq 1.0)$, and $\mu \mathrm{G}$ magnetic fields over very large volumes (halo diameters are often $\geq 1 \mathrm{Mpc}$ ). These diffuse radio sources are not associated with any individual galaxy but are truly a cluster phenomenon. They are generally found in very rich (Abell richness class $\geq 2$ ), X-ray luminous clusters. Some form of in-situ particle acceleration and magnetic field amplification is required to maintain the synchrotron emission on such large scales. Turbulent galaxy wakes have insufficient kinetic energy to power the radio halos (e.g. De Young 1992).

Recently, it has been found that most radio halos appear to lie in clusters that are undergoing cluster-cluster mergers (e.g., Coma, A2255, A2256, A3667) (e.g. Burns 1998). This seems to suggest that such mergers play an integral role in the formation and evolution of the radio halos. Cluster mergers do have sufficient kinetic power, comparable to the total cluster thermal energy loss rate $\left(10^{46} \mathrm{ergs} / \mathrm{sec}\right)$, to energize halos with typical radio luminosities of $\sim 10^{42}$ ergs/sec. 


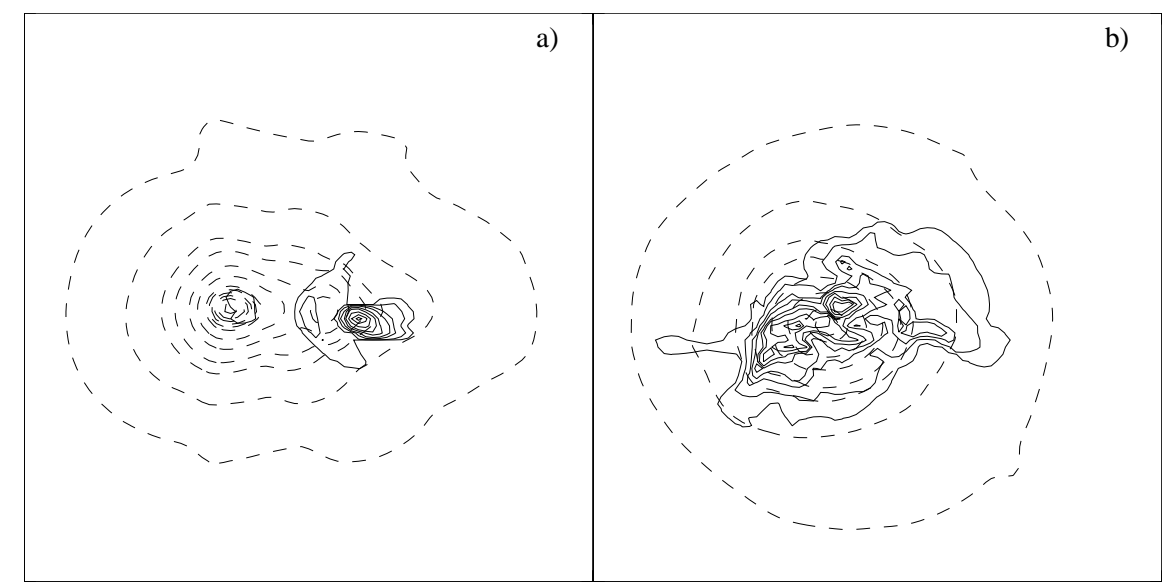

Fig. 3. Preliminary results from a numerical simulation of radio halo evolution, a) 0.4 Gyr before core passage and b) 6 Gyrs after core passage. Dashed contours represent the soft x-ray emitting thermal ICM while the solid contours represent the sychrotron emission from the relativistic plasma. Each panel is $\sim 6 \mathrm{Mpc}$ on a side.

One of us (Roettiger) has developed a new N-body/MHD code for modeling the evolution of magnetic fields during cluster mergers (Roettiger, Stone, \& Burns 1999). The ICM and B-fields are evolved using the Eulerian, finite difference code ZEUS and the particles are evolved using a PM (particle-mesh) algorithm. A diffusive shock acceleration model, whereby the shock strength determines the power-law slope of the injected particle energy spectrum, was used to produce simulations of radio emission during cluster mergers. This technique was applied to Abell 3667 and found to reproduce the observed large scale radio "arches" (Röttgering et al. 1997), which we identify as the sites of shocks formed during the merger between clusters (Roettiger, Burns, \& Stone 1999). The numerical model also reproduces the elongated cluster x-ray emission and is consistent with the observed bimodal distribution of cluster galaxies in A3667.

We have now extended these numerical simulations to include more sophisticated diffusive shock and turbulent particle acceleration along with synchrotron aging following the approach of Jun \& Jones (1999). This technique treats the relativistic electrons as multiple fluid components each representing a range in particle momenta. In Fig. 3, we show preliminary results of the evolution of the radio and x-ray emission during a cluster-cluster merger. Lending support to the evolutionary scenario suggested in Roettiger, Stone, \& Burns (1999), these new simulations show the radio emission in early epochs to be dominated by shock acceleration producing edge-brightened, off-center relics resembling those in A2256 and A3667. At later times, the shocks diminish in strength as the cluster relaxes and turbulence drives the particle acceleration producing center-filled halos that resemble Coma. 


\section{A Possible Radio Analog of the Butcher-Oemler Effect}

A final possible manifestation of accretion and mergers in the cluster environment on radio sources is illustrated using new observations of weak radio galaxy emission in more distant rich clusters. We used the VLA to deeply image $z>0.2$ very rich clusters (Abell richness class $\geq 2$ ) down to 20 -cm radio powers of $\approx 10^{21.8} \mathrm{~W} / \mathrm{Hz}$. At such low powers, the radio luminosity function (RLF) is dominated by star formation and weak AGNs. Each of these clusters was also detected by the ROSAT all-sky survey at $0.5-2.0 \mathrm{keV}$ energies.

At 20 -cm powers $\geq 10^{23} \mathrm{~W} / \mathrm{Hz}$, we find that the RLF and the spatial distribution of radio sources look virtually identical between samples of nearby $(z \leq 0.09)$ and more distant $(z=0.2-0.4)$ rich clusters. These sources are principally traditional AGNs that are associated with galaxies generally concentrated within the cores of clusters. Stocke et al. (1999) confirms this lack of change in the characteristics of powerful radio sources in even more distant clusters selected from the Einstein medium sensitivity x-ray survey. Thus, in spite of the dramatic evolution of the cluster environment, the distribution and luminosities of powerful radio sources remain relatively unaffected by these events.

On the other hand, there appear to be some very interesting, significant changes in the population of low power radio sources over these same redshift intervals. For radio sources with $P_{20 \mathrm{~cm}} \leq 10^{23} \mathrm{~W} / \mathrm{Hz}$, the spatial distribution of weak radio galaxies is much broader extending over several Mpcs rather than being confined to the cluster core (several hundred kpc) (e.g. Morrison 1999, Rizza et al. 2000). This extent is more on the supercluster than the cluster scale. Furthermore, in the case of some individual clusters such as A2125, the number of weak radio sources is much greater (factor of 5) than that in comparable richness nearby clusters (Owen et al. 1999). There is also some suggestion that this trend of increased numbers of weak radio sources becomes stronger at higher redshifts $(z \sim 0.4)$ (Morrison 1999).

These low power radio sources are likely to be starburst galaxies. Such starburst emission may be triggered as groups of galaxies accrete onto richer clusters. The x-ray emission observed by ROSAT for many of these clusters is clumpy and suggests that on-going mergers are common at these epochs (Rizza et al. 1999), as suggested by the numerical simulations. Thus, we may be seeing a radio analog of the Butcher-Oemler effect in these rich clusters. 


\section{Conclusions}

The gaseous environment within groups and clusters of galaxies is filled with "stormy weather" including shocks, high winds, and turbulence. This violent weather is produced by mergers between clusters and groups, and by on-going accretion of matter from supercluster filaments that connect clusters to the large-scale structure of the universe. New AMR numerical simulations reveal great complexity within the ICM that has important consequences for the extended radio sources that lie within clusters.

Wide-angle tailed radio sources are likely bent into a $\mathrm{V}$-shape as the result of bulk flows of gas moving by the central cluster radio galaxies at velocities of $\geq 1000 \mathrm{~km} / \mathrm{sec}$. This dynamic pressure arises from mergers and accretion of gas from large-scale filaments. The observed correlations between x-ray elongations of the ICM with the WAT radio tail directions, and between the supercluster axis and the tail directions support this hypothesis.

New N-body/MHD simulations, which include diffusive shock and turbulent acceleration of relativistic electrons, are able to reproduce the morphologies of radio halos resulting from mergers between clusters. A possible evolutionary scenario is suggested whereby early-stage mergers produce halos whose morphologies are dominated by the effects of shocks (e.g., A3667) whereas late-stage mergers produce center-filled halos dominated by turbulence (e.g., Coma).

Finally, we have observed strong evolution in a population of weak radio sources in distant rich clusters. These weak sources are likely to be starburst galaxies. The x-ray emission from these clusters is often asymmetrical and clumpy. This suggests that the starbursts may be stimulated by galaxies in groups infalling from supercluster filaments onto rich clusters, similar to what has been proposed to explain the excess of blue galaxies in more distant clusters (i.e., Butcher- Oemler effect).

We thank Michael Ledlow, Anatoly Klypin, and Wolfgang Voges for their collaboration on aspects of this research. This work was supported by the NSF (AST-9896039) and by NASA.

\section{References}

Burns, J. O., 1998, Science, 280, 400.

De Young, D. S., 1992, ApJ, 386, 464.

Eilek, J. A., Burns, J. O., O'Dea, C. P., and Owen, F. N., 1984, ApJ, 278, 37. 
Giovannini, G., Tordi, M., and Feretti, L. 1999, New Astronomy, in press. (astro$\mathrm{ph} / 9904210)$

Gómez, P. L., Pinkney, J., Burns, J. O., Wang, Q., Owen, F. N., and Voges, W., 1997, ApJ, 474, 580.

Jun, B.-I., and Jones, T., 1999, ApJ, 511, 774.

Loken, C. et al. 1999, in preparation.

Morrison, G., 1999, Ph.D. dissertation, University of New Mexico.

Navarro, J. F., Frenk, C. S., and White, S. D. M., 1997, ApJ, 490, 493.

Novikov, D. I. et al., 1999, MNRAS, 304, L5.

Owen, F. N., Ledlow, M., Keel, W., and Morrison, G., 1999, astro-ph/9905004.

Pinkney, J. et al., 1993, ApJ, 416, 36.

Pinkney, J. et al., 1999, in preparation.

Rizza, E., Burns, J. O., Ledlow, M., Owen, F., Voges, W., and Bliton, M., 1999, MNRAS, 301, 328.

Rizza, E. et al. 2000, in preparation.

Roettiger, K., Burns, J. O., and Loken, C., 1996, ApJ, 473, 651.

Roettiger, K., Stone, J. M., and Burns, J. O., 1999, ApJ, 518, 594.

Roettiger, K., Burns, J. O., and Stone, J. M., 1999, ApJ, 518, 603.

Röttgering, H., Wieringa, M., Hunstead, R., and Ekers, R. 1997, MNRAS, 290, 577

Stocke, J. T., Perlman, E., Gioia, I., and Harvanek, M., 1999, AJ, 117, 1967. 